\section{Fight over CRISPR IP flares up}

The dispute over who was first to invent CRISPR-Cas9 genome editing in eukaryotic cells has resumed with the US Patent and Trademark Office (USPTO) declaring an interference between the University of California (UC) and the Broad Institute of MIT and Harvard. The patent office's decision came in June, just nine months after a federal court upheld a no-interference judgement that gave the Broad control of key CRISPR intellectual property. The new interference case involves 13 of the Broad's 15 patents and one application, and 10 patent applications submitted by UC Berkeley and partners. It is a signal that the USPTO has determined that one or more of Berkeley's patent applications are substantially the same as previously issued Broad patents. Since the federal court's September 2018 ruling, which declared no interference and appeared to be a win for Broad and Harvard, UC Berkeley has been granted six CRISPR patents in the United States. These include a foundational patent that covers systems and methods for the use of single-molecule guide RNAs combined with the Cas 9 protein to modify target DNA in any setting, including in vitro and in cells. In addition, in May the Broad Institute had its third European CRISPR patent revoked by the European Patent Office for lacking in inventive step. UC Berkeley has maintained a dominant CRISPR patent position in Europe, while the Broad has held the upper hand in the United States. However, the newly filed interference could upend the current balance of power. "The initiation of this interference proceeding highlights that previous decisions involving the Broad did not determine who was the first to invent this technology, and it lays out a pathway for resolving this important issue," said Eldora Ellison, lead patent strategist on CRISPR matters for UC Berkeley.

Published online: 4 September 2019 https://doi.org/10.1038/s41587-019-0256-2

\footnotetext{
C "It's hard to say for sure what we can achieve in the next few decades, but I think with CRISPR we have a chance to catch up to climate change." Yiping Qi, a plant genome engineer at the University of Maryland, comments on how CRISPR could accelerate the introduction of plants that withstand droughts, heat waves, megafloods and other expected climate changes. (Wired, 8 August 2019)
}

mainly to the lymphodepletion regimen and the interleukin-2 infused to boost TIL survival and growth, these resolved two weeks after infusion. The $38 \%$ response rate was, "an excellent result in this population," commented immunologist Ignacio Melero of the Clinica Universidad de Navarra in Madrid, Spain at ASCO. At an 8.8-month follow up, 17 of 25 responders had an ongoing response, suggesting durable effects.

"My concern is that with more follow up their duration of response may go down," says Bernatchez. "But right now they're showing pretty good numbers."

Iovance is now enrolling a new melanoma cohort using its secondgeneration TIL protocol, which cuts the previous five- to six-week manufacturing time to 22 days. Although Iovance has not disclosed the details for this improved protocol, Iovance's Fardis expects trial enrollment to be complete by the first quarter of next year, with a possible Biologic License Application filing by year's end.

At ASCO, Iovance also reported ongoing results for LN-145 in its open-label phase 2 trial for metastatic cervical cancer. So far 12 of 27 patients have experienced an objective response, including three complete responses. Ten of those whose disease responded had an ongoing response at a median follow up time of 7.4 months. That's impressive, says Bernatchez, but "I'd be careful with those high response rates," she adds. "I won't believe it until I see that the response rates are really durable." Given the FDA's willingness to consider the complete data, Fardis says Iovance may file its Biologic License Application for cervical cancer by late 2020 .

For companies, the number of TILs needed for infusion poses a greater logistical challenge than for CAR-Ts. A single CAR-T dose is around 100 million cells. "TIL [therapy] (as best we know now) requires products with 100 times that many cells, on the order of 30-50 billion," writes Goff in an e-mail. "Cryopreserving that many cells and maintaining viability of the thawed product is certain to be a challenge."

Even with manufacturing obstacles cleared, there's still a huge gap between success in melanoma and cervical cancer, on the one hand, and other cancers on the other. This may be because breast, colon, ovary, prostate and pancreatic cancers are poorly immunogenic. Of the harvested TILs, only a fraction are reactive against the tumors, so giving patients their own heterogeneous TILs appears futile. "Unselected TILs in our hands certainly did not work in patients with epithelial cancers," says Goff. "We had zero responses."

To boost tumor killing, Rosenberg's group selects only tumor neoantigen-reactive
T cells among patients' TILs. First they isolate tumor DNA or RNA, and use whole exome or transcriptome sequencing to identify all amino acid-altering mutations by comparison with sequenced normal tissue. Then the NCI team designs gene constructs containing sequences for all the mutant peptides that could be displayed on the major histocompatibility complex (MHC) of antigen-presenting cells. They then string sequences together into 'tandem minigenes' and insert them into the patient's own antigen-presenting cells, to test patient $\mathrm{T}$ cells for reactivity. The reactive $\mathrm{T}$ cells, expanded in culture, are reinfused into the patient.

\section{"At some point we will get there... right now, we should rely on the patient's immune system, which has selected the right TILs:"}

The main advantage of the NCI selection method is exquisite specificity for a given patient's tumor. With the tandem mini-genes approach, the NCI has achieved a roughly $15 \%$ clinical response rate across metastatic epithelial cancers. This includes the breast cancer patient with the ongoing complete response that Goff highlighted at ASCO.

But to design and construct the tandem minigenes alone takes up to a month. The whole process, from tumor excision to product readiness, takes a median of ten weeks, says Goff. That's not fast enough for most cancer patients, says Bernatchez. "I think [the NCI approach] has value, but unless it's highly streamlined, it's going to be very difficult to apply to large patient populations," she says. "You need to get it at least under one month."

Iovance's Fardis agrees. "We have to think about a process that can be industrialized," she says. Iovance shaved its process down to 22 days partly by omitting the neoantigen-reactive T-cell selection step. Such selection isn't necessary, Fardis says. "Science right now is not ready to collect all the known neoantigens," she says. (The NCI system misses post-translational modifications and edited RNA.) "At some point we will get there ... right now, we should rely on the patient's immune system, which has selected the right TILs," although in diluted form.

So for now, both Iovance and MD Anderson are using bulk, unselected TILs. But they're exploring markers on T cells that could enable selection of tumor-reactive $\mathrm{T}$ cells, as well as ways of making $\mathrm{T}$ cells more potent. Iovance hopes to have a selective T-cell product in the clinic by 2020 . "I don't 